\title{
Daño renal asociado a metales pesados: trabajo de revisión
}

\section{Renal damage associated with heavy metals: review work}

\author{
Juan Daniel Díaz García ${ }^{1, *}$, Emmanuel Arceo ${ }^{2}$ \\ ${ }^{1}$ Departamento de Nefrología y Metabolismo Mineral del Instituto Nacional de Ciencias Médicas y Nutrición "Salvador Zubirán”, Ciudad de México, México \\ ${ }^{2}$ Departamento de Nefrología del Hospital General de México "Eduardo Liceaga". Ciudad de México, México
}

\begin{abstract}
Resumen
La enfermedad renal crónica (ERC) representa un importante problema de salud en todo el globo. Actualmente, es esencial para su prevención el conocimiento de los factores ambientales asociados con la enfermedad. Se reconocen diversos metales pesados, entre los que destacan el cadmio $(\mathrm{Cd})$, plomo $(\mathrm{Pb})$, arsénico $(\mathrm{As})$ y mercurio $(\mathrm{Hg})$, que están claramente asociados con la lesión renal y la progresión de la ERC. Estudios en animales y humanos demuestran, principalmente, una clara asociación entre la exposición a estos metales y la presencia de daño renal crónico, donde la fisiopatología de cada uno de ellos es importante para entender el mecanismo de daño renal. La presente revisión tiene como objetivo analizar, tanto la fisiopatología y manifestaciones clínicas de la nefrotoxicidad asociada a dichos metales, como los diferentes estudios que se han realizado en humanos y animales.
\end{abstract}

Palabras clave: nefrotoxicidad, cadmio, plomo, arsénico, mercurio, necrosis tubular aguda, enfermedad renal crónica, metales pesados.

doi: http://dx.doi.org/10.22265/acnef.5.2.254

\begin{abstract}
Catheter-associated infection is an entity with multiple complications, a condition that alters the quality of life of all patients Chronic kidney disease (CKD) represents an important health problem in the entire global population, where knowledge of the environmental factors associated with this disease is currently essential for its prevention. Several heavy metals are recognized, including Cadmium $(\mathrm{Cd})$, Lead $(\mathrm{Pb})$, Arsenic $(\mathrm{As})$ and Mercury $(\mathrm{Hg})$, which are clearly associated with renal damage and progression of CKD. Studies in animals and humans primarily demonstrate a clear association between exposure to these metals and the presence of chronic renal damage, where the pathophysiology of each of these metals is important in understanding the mechanism of renal damage. The present review aims to analyze the pathophysiology and clinical manifestations of the nephrotoxicity associated with these metals, as well as the different studies in both humans and animals that have been performed.
\end{abstract}

Key words: Nephrotoxicity, cadmium, lead, arsenic, mercury, acute tubular necrosis, chronic kidney disease, heavy metals.

doi: http://dx.doi.org/10.22265/acnef.5.2.254

Referenciar este artículo: Díaz Gacía JD, Arceo E. Rev. Colomb. Nefrol. 2018;5(1): 43-53 doi: http://dx.doi.org/10.22265/acnef.5.2.254

*Correspondencia: Juan Daniel Díaz García: judan digar@hotmail.com

Recibido: $17-04-17 \cdot$ Aceptado: $21-08-17 \cdot$ Publicado en línea: 23-11-17 


\section{Introducción}

$\mathrm{L}$ a enfermedad renal crónica (ERC) es un problema de salud pública mundial con alta prevalencia. La identificación de los diversos factores de riesgo relacionados con ella es primordial para prevenir el desarrollo y el crecimiento del fenómeno. ERC es, en términos generales, el resultado de diversas alteraciones heterogéneas que alteran la estructura y función renal. La variación de la expresión de la enfermedad está directamente relacionada con patologías previas, lesión estructural, grado de severidad y tiempo de progresión.

Por medio de estudios epidemiológicos, se ha demostrado la asociación entre la exposición al medio ambiente y el desarrollo y progresión de esta enfermedad.

Algunas formas de ERC se han relacionado con nefrotoxinas ambientales, tales como exposición a metales pesados, micotoxinas producidas por hongos en alimentos almacenados, contaminantes del aire y plaguicidas ${ }^{1}$.

En los últimos 50 años, el estudio de los efectos tóxicos de metales pesados en el ser humano ha cobrado particular importancia, debido a que grandes cantidades de estos productos, desechadas como parte de la actividad industrial, no son biodegradables y persisten en el medio ambiente durante largos períodos de tiempo.

La exposición al cadmio, al plomo y, en menor medida, al mercurio constituyen una preocupación particular, ya que muchos estudios epidemiológicos han mostrado una fuerte asociación entre la exposición a estos metales pesados, los marcadores de daño renal y la progresión de la ERC. La exposición al arsénico sigue siendo un importante problema de salud pública, ya que millones de personas están expuestas a fuentes de agua con niveles por encima del límite ${ }^{2}$.

\section{Metodología}

Para evaluar el papel de estos contaminantes en la enfermedad renal, se realizó un análisis con base en estudios sobre la fisiopatología, mecanismos de daño renal y trabajos en humanos y animales. Todos estos, asociados a la exposición de dichos metales pesados.

Se llevaron a cabo búsquedas en PubMed (http:// www.ncbi.nlm.nih.gov/pubmed), para encontrar todos los estudios publicados que evalúan la relación entre la enfermedad renal y la exposición al cadmio, arsénico, plomo y mercurio.

\section{Fisiopatología y mecanismo de daño renal}

Los mecanismos fisiopatológicos de daño renal por los diferentes contaminantes ambientales son complejos. No se conocen por completo varios aspectos relacionados con su metabolismo y mecanismo de daño en el organismo. Para entender sus efectos nefrotóxicos, se analizarán resumidamente estos mecanismos por cadmio $(\mathrm{Cd})$, plomo $(\mathrm{Pb})$, arsénico $(\mathrm{As})$ y mercurio $(\mathrm{Hg})$ (Tabla 1).

Tabla 1. Mecanismo de daño por intoxicación con metales pesados en animales.

\begin{tabular}{|l|l|l|}
\hline \multicolumn{1}{|c|}{ Contaminante } & Vía de entrada & \multicolumn{1}{c|}{ Mecanismo de daño } \\
Cadmio & Vía gástrica & $\begin{array}{l}\text { - Apoptosis: activación por vía de las Caspasas. } \\
\text { - Inhibición de la ECA. } \\
\text { - Disminución de producción de NO. }\end{array}$ \\
\hline Dióxido de azufre & Vía respiratoria & - Daño en ADN multiorgánico. \\
\hline Arsenato & Vía respiratoria & - Alteración del transporte de sodio, fosfato, glucosa, y PHA. \\
\hline
\end{tabular}

ECA:enzima convertidora de angiotensina, NO: Óxido nítrico, ADN: ácido desoxirribonucleico, PHA: Paraaminohiputaro. 
El cadmio que se ingiere se encuentra unido a las proteínas metalotioneína (MT) y fitoquelatina, que son catalizadas por el ácido clorhídrico $(\mathrm{HCl})$ estomacal, liberando el $\mathrm{Cd}$ para su absorción en yeyuno-íleon por los transportadores DMT1 (transportador de metales divalentes 1) y ZIP-8 (proteína transportadora de Zinc 8) ) $^{3,4}$. En el torrente sanguíneo se une a albúmina y es transportado al hígado, donde se une al glutatión (GSH) y a la metalotioneína-1 (MT1). El complejo Cd-MT1 es secretado en la bilis; sin embargo, regresa al torrente sanguíneo por la circulación enterohepática.

Dentro de las células del túbulo contorneado proximal(TCP), el complejo Cd-MT1 es almacenado y degradado en los lisosomas. Posteriormente, el Cd libre es transportado hacia el citoplasma por el DMT-1 lisosomal ${ }^{5}$. La activación de la proteína cinasa $\mathrm{C}$ incrementa la expresión del transportador DMT-1 y, por lo tanto, la toxicidad tubular del $\mathrm{Cd}^{6}$. El Cd libre es acumulado en la mitocondria, inhibiendo la cadena respiratoria en el complejo III. Esto resulta en disfunción mitocondrial y formación de radicales libres, lo cual activa la vía de caspasas con subsecuente apoptosis. Además, se une a los grupos sulfhidrilo de las proteínas creando su desnaturalización (Figura 1).

Se ha demostrado que el $\mathrm{Cd}$ interfiere con las actividades enzimáticas del complejo calcio / calmodulina, que inhibe la acción de la Na-KATPasa y que estimula la actividad de las MAPK (proteínas quinasas activadas por mitógenos). En las uniones paracelulares, afecta la distribución de las proteínas de la unión paracelular y disminuye la resistencia transepitelial ${ }^{7,8}$.

Otro mecanismo de nefrotoxicidad es aquel mediado por la formación de anticuerpos a la MT. $\mathrm{La}$ exposición al $\mathrm{Cd}$ incrementa la producción de MT en el hígado y el riñón, lo que constituye un mecanismo de defensa para limitar su toxicidad. Sin embargo, una vez que se excede la capacidad de las MT de almacenar Cd, este, en forma libre, induce la formación de anti-MT, que también son tóxicos para las células del TCP 9 .

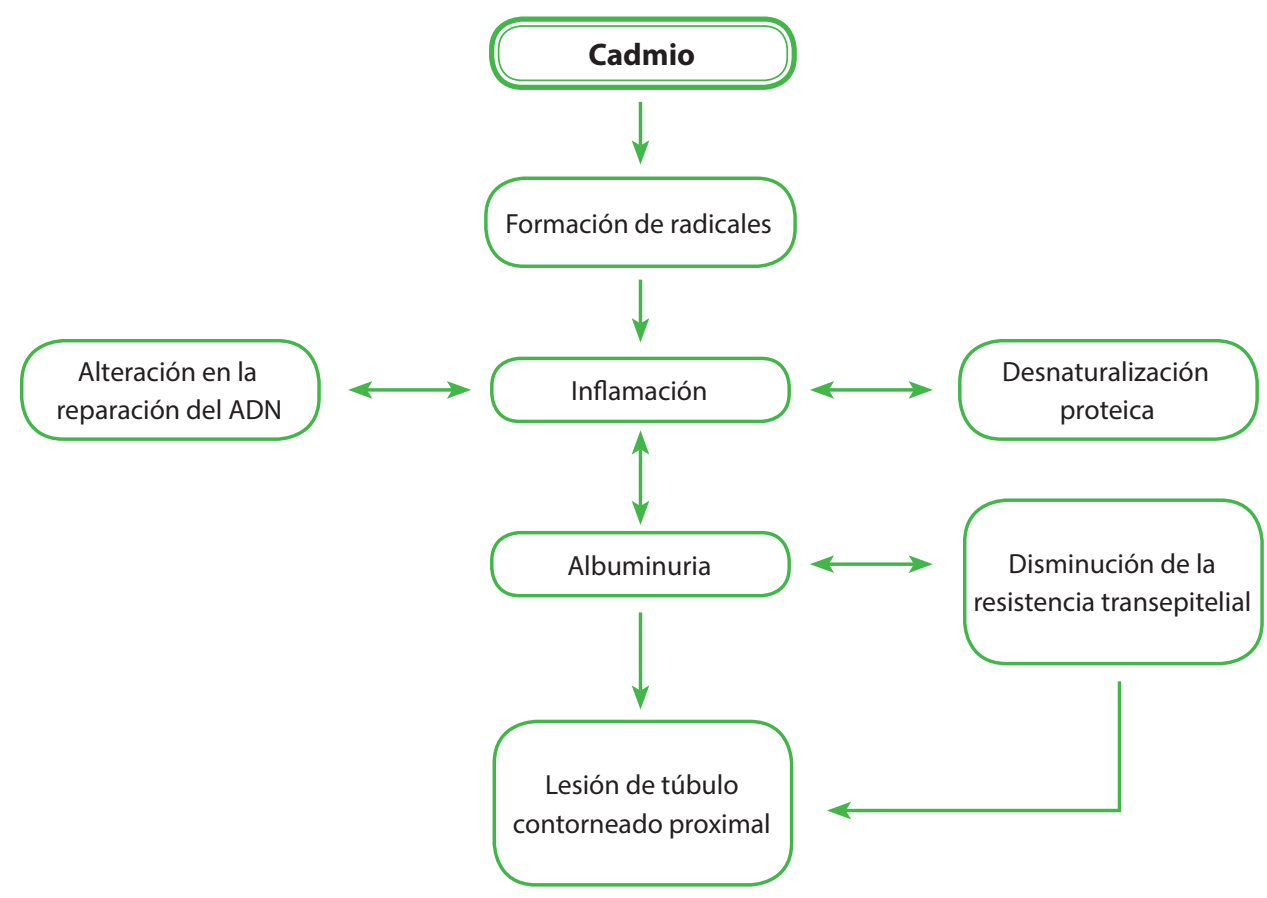

Figura 1. Fisiopatología y mecanismo de daño renal del cadmio. 
El plomo se absorbe, principalmente, por ingesta e inhalación y, en menor medida, a través de la piel. La absorción intestinal es mediada por el transportador DMT-1 y se incrementa con la ingesta deficiente de hierro $(\mathrm{Fe})$ y zinc $(\mathrm{Zn})$. Una vez en la sangre, el $99 \%$ del $\mathrm{Pb}$ se une a proteínas del hematíe y es distribuido a tejidos blandos y hueso. Este es el principal reservorio de $\mathrm{Pb}$ en el organismo, y en períodos de remodelación ósea, como la adolescencia y el embarazo, el paso de $\mathrm{Pb}$ a la sangre se incrementa ${ }^{10}$.

$\mathrm{E} 1 \mathrm{~Pb}$, unido a proteínas de bajo peso molecular $(<$ del $1 \%$ del total $)$, se filtra libremente a través del glomérulo y es reabsorbido por las células del TCP por el proceso de endocitosis. De forma intracelular, el $\mathrm{Pb}$ causa daño mitocondrial, formación de radicales libres, depleción intracelular de GSH y apoptosis ${ }^{9}$. El $\mathrm{Pb}$ afecta reacciones enzimáticas en las que interviene el calcio, incluso el receptor sensor de calcio puede ser activado por $\mathrm{Pb}$, lo cual sugiere otros mecanismos de nefrotoxicidad por este metal ${ }^{10,11}$. En el organismo, la excreción urinaria es la principal vía de pérdida de $\mathrm{Pb}$.

$\mathrm{El} \mathrm{Pb}$ induce la activación del factor de transcripción nuclear- $\kappa \beta$, la activación del sistema intrarrenal de renina-angiotensina y la atracción de macrófagos. Esto genera un proceso inflamatorio en el intersticio renal, que podría estar implicado en el desarrollo de daño al tubulointersticio y en la hipertensión arterial ${ }^{12}$.

En células endoteliales, se ha demostrado que el incremento en la formación de radicales libres por $\mathrm{Pb}$ disminuye la producción de óxido nítrico y la expresión de la enzima guanilato-ciclasa. Esto permite explicar la patogénesis de la hipertensión arterial inducida por este metal ${ }^{11-16}$. Además, estimula la actividad del NADPH oxidasa incrementando la producción de superóxido y peróxido de hidrógeno, lo que afecta al estrés oxidativo y el potencial óxidoreducción intracelular.

El arsénico se absorbe por ingesta, inhalación $\mathrm{y}$, en menores cantidades, por la piel. Una vez absorbido, se distribuye por todos los tejidos del cuerpo. La ingesta de selenio y vitamina B disminuye la absorción intestinal de As. En el hígado es metilado en un proceso mediado por el GSH, lo cual disminuye su toxicidad y facilita su excreción biliar y urinaria ${ }^{17}$.

El As ingresa al interior de la célula a través de las aquagliceroporinas AQ3 y AQ9. En el hígado, esta última es importante para la excreción biliar de $\mathrm{As}^{18}$.

Los MRP-1 y 2 (ATP binding cassette-multidrug resistance protein) son otro grupo de proteínas transportadoras de As en hígado, donde transportan elAs, conjugado con GSH, a la bilis. El transportador MRP-2 también se localiza en las células del túbulo proximal, lo que favorece la introducción de As en estas células ${ }^{19}$. La toxicidad de As en las células del TCP se debe a la depleción de GSH y al incremento en la actividad oxidativa de los radicales libres (Figura 2).

El mercurio provoca toxicidad al organismo a través de la piel, inhalación o ingestión. El Hg tiene gran afinidad por el tejido renal. La nefrotoxicidad comúnmente se manifiesta como síndrome nefrótico y lesión tubular ${ }^{20,21}$. El síndrome nefrótico asociado a toxicidad por $\mathrm{Hg}$ es reversible después de su completa eliminación.

El $\mathrm{Hg}$ induce nefropatía membranosa a largo plazo, debido a sus pequeñas concentraciones en cosméticos y contacto ocupacional. El mecanismo de daño renal que condiciona glomerulopatía por $\mathrm{Hg}$ no está bien esclarecido. Se ha sugerido que el síndrome nefrótico posterior a la exposición con $\mathrm{Hg}$ es una reacción inmune antígeno-anticuerpo inespecífica a metales pesados. El cloruro mercúrico $\mathrm{y}$ el monometillmercurio inhiben la función de los linfocitos humanos, incluidos proliferación, expresión de las células activadas por marcadores de superficie y producción de citocinas ${ }^{22-26}$.

\section{Estudios en animales}

Varios estudios realizados en animales demuestran la asociación entre la exposición de los diferentes contaminantes ambientales y sus efectos nefrotóxicos. 


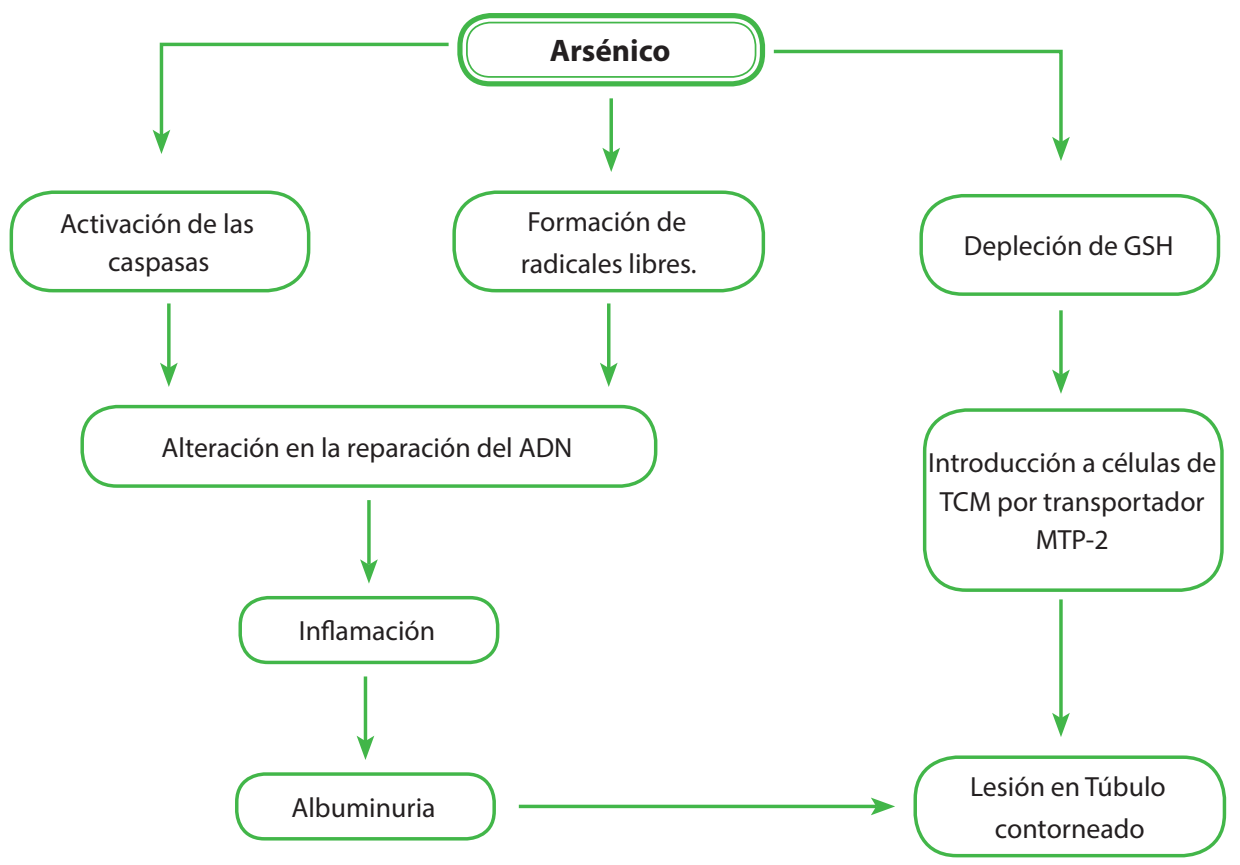

Figura 2. Fisiopatología y mecanismo de daño renal del arsénico.

Con respecto al cadmio, en un modelo experimental de células mesangiales de rata, se indujo apoptosis celular por medio de la vía extrínseca o la vía mediada por la mitocondria (intrínseca) y se encontró que la camptotecina inicia la vía intrínseca, con la activación de la caspasa 9 y la escisión de la pro-caspasa 3. Allí, el factor de necrosis tumoral da lugar a la actividad de la caspasa 8 y a la escisión de la pro-caspasa 3 como punto de convergencia de las dos vías. Tal estudio concluye que la concentración de $10 \mu \mathrm{M}$ de $\mathrm{Cd}$ puede prevenir la fragmentación de ADN (ácido desoxirribonucleico), demostrando que el $\mathrm{Cd}$ es anti-apoptótico en las células mesangiales de rata, actuando por un mecanismo que puede, en general, inhibir las caspasas ${ }^{27}$. En otro estudio, se encontró que el $\mathrm{Cd}$ inhibe la enzima convertidora de angiotensina (ECA) a baja, media y alta dosis sin efecto dosis-respuesta en ratas con hipertensión paradójicamente inducida. Se postuló que los efectos vasculares del $\mathrm{Cd}$ predominaron sobre sus efectos centrales en las ratas hipertensas ${ }^{28}$.

Se ha investigado, también, el papel de la molécula de lesión renal 1 (Kim-1), una glicoproteína transmembrana que se excreta en la orina después de una lesión por exposición a un nefrotóxico, particularmente el cadmio (Cd). En un estudio, se utilizó Kim-1 como biomarcador para determinar la nefrotoxicidad del $\mathrm{Cd}$ en ratones machos. En el estudio, se administró $\mathrm{Cd}$ a diario durante doce semanas, determinado semanalmente en orina Kim-1, proteínas, creatinina, metalotioneínas y proteínas de culuas claras (CC-16). Se detectaron niveles significativos de Kim-1 en la orina a las seis semanas, que continuaron aumentando a lo largo del período de tratamiento. La aparición de Kim-1 se produjo de cuatro a cinco semanas antes de la aparición de proteinuria y de una a tres semanas antes de la aparición de la metalotioneína y CC-16. Sus resultados sugieren que se utilice Kim-1 como biomarcador en los estadios tempranos, en la lesión inducida por $\mathrm{Cd}$ en los túbulos proximales ${ }^{29}$.

Al estudiar el dióxido de azufre (SO2) como contaminante ambiental, se ha demostrado que, en ratones, la exposición al mismo causa daño en el ADN de múltiples órganos y que los derivados del SO2 son agentes que dañan el ADN sistémico. Se sugiere que la exposición a $\mathrm{SO} 2$ tiene un riesgo 
potencial de daño para el ADN en múltiples órganos de mamíferos y podría estar relacionado con la carcinogénesis o mutación del ADN genómico ${ }^{30}$.

Se ha notado que el arsenato actúa a nivel del transporte de sodio, fosfato, glucosa, y paraaminohiputaro (PHA), así como en el metabolismo oxidativo en el túbulo contorneado proximal de riñones de conejos y reduce el transporte neto de sodio y fosfato. El transporte de glucosa se ve inhibido ligeramente, mientras que la secreción de PHA no se afecta ${ }^{31}$.

El monóxido de carbón ( $\mathrm{CO})$ se ha estudiado en riñones de ratones, donde se observó que dicho gas aumentaba al administrar 5-aminolevulínico ferroso (5-ALA/Fe2) y, en sangre, disminuían las concentraciones máximas de creatinina y nitrógeno ureico. Los ratones tratados con 5-ALA/Fe2, disminuían significativamente el daño tubular y el número de células apoptóticas. Estos hallazgos sugieren que 5-ALA/Fe2 protegen el riñón de la lesión por reperfusión en la isquemia renal, al reducir la infiltración por macrófagos y al disminuir la apoptosis celular renal mediante la generación de $\mathrm{CO}^{32}$.

\section{Estudios en humanos}

En diversos estudios epidemiológicos se ha postulado la asociación entre exposición al arsénico (As) e hipertensión arterial y otras enfermedades cardiovasculare $^{33}$. En estudios de tipo experimental fueron expuestos a altas dosis de este compuesto. En un estudio epidemiológico basado en información pasiva en Utah, Lewis et al. ${ }^{34}$ encontraron incremento en la tasa de mortalidad por enfermedad renal en hombres, no así en mujeres, por exposición de altas concentraciones de As. Meliker et al. ${ }^{35}$ encontraron que el As en agua potable excede los 200-300 $\mathrm{mg} / \mathrm{L}$, se asocia con elevadas tasas de mortalidad de diabetes mellitus, enfermedad cerebrovascular y enfermedad renal en hombres y mujeres.

Hsueh et al. ${ }^{36}$ estudiaron a 125 personas, en Taiwán, con tasa de filtrado glomerular $(\mathrm{TFG})<60$ $\mathrm{ml} / \mathrm{min}$ y 229 personas con función renal normal. Encontraron una débil asociación entre los niveles urinarios de As y la disminución de la función renal $(r 2=0.04, p \leq 0.001)$. En este estudio, la alta excreción renal de As se vinculó con un menor nivel de licopeno en plasma, mostrando que el daño renal estaba medido por incremento del estrés oxidativo.

El As es promotor de inflamación, estrés oxidativo y disfunción endotelial por distintos mecanismos, incluida la activación de transcripción de factores como proteína-1 y el factor nuclear $\kappa \beta^{37-}$ ${ }^{40}$. Los mecanismos biológicos responsables de la diabetes mellitus inducida por As permanecen, en gran parte, desconocidos. Evidencia reciente sugiere que los arsenicales trivalentes pueden suprimir el estímulo insulínico para la captación de glucosa, al interferir con la movilización de los transportadores de glucosa en las células adiposas ${ }^{41}$. Pueden, también, interferir con los factores de transcripción involucrados en la expresión del gen relacionado con la insulina ${ }^{42}$.

Para evaluar su nefrotoxicidad, se han realizado diversos estudios en poblaciones expuestas a $\mathrm{Cd}$, con diferentes grados de sensibilidad e importancia clínica. Entre estos, la albúmina urinaria elevada, en ausencia de aumento en la excreción de proteínas de bajo peso molecular, es un indicador temprano de daño glomerular y se observa en concentraciones de 3,6 a 4,2 $\mu \mathrm{g} \mathrm{Cd} / \mathrm{g}$ de creatinina en orina ${ }^{43-44}$. La proteinuria de bajo peso molecular en personas expuestas a $>10 \mu \mathrm{g} \mathrm{Cd} / \mathrm{g}$ creatinina en orina es irreversible y exacerba el declive relacionado con la edad y $\mathrm{TFG}^{45-47}$. La elevación de las enzimas $\mathrm{N}$-acetil- $\beta$-D-glucosaminidasa (NAG) y alaninaaminopeptidasa (AAP), se han asociado a daño tubular renal con exposiciones ocupacionales de $\mathrm{Cd}$ de 3,7 a $6,3 \mathrm{mg} \mathrm{Cd} / \mathrm{g}$ creatinina en orina ${ }^{44,45,48}$.

Liang et al. ${ }^{49}$ demostraron que la exposición al bajo nivel de plomo $(\mathrm{Pb})$ ambiental puede acelerar la insuficiencia renal progresiva en pacientes sin diabetes, pero que padecen enfermedad renal crónica. Los autores observaron a 202 pacientes con insuficiencia renal crónica durante 24 meses. Posteriormente, 64 sujetos con niveles elevados de $\mathrm{Pb}$ en sangre fueron divididos, aleatoriamente, en dos grupos. Durante tres meses, los pacientes del grupo 'de quelación' recibieron terapia con ácido 
etildiaminotetraacético disódico de calcio (EDTA). Por su parte, los del grupo 'control' recibieron placebo. Con base en sus resultados sugieren que la terapia de quelación repetida puede mejorar la función renal y retrasar la progresión de la insuficiencia renal.

Sommar et al. ${ }^{50}$ realizaron un estudio donde correlacionan $\mathrm{Pb}, \mathrm{Hg}$ y $\mathrm{Cd}$ con ERC. Analizaron muestras de 118 pacientes para determinar las concentraciones de $\mathrm{Cd}, \mathrm{Pb}$ y $\mathrm{Hg}$. Realizando un análisis prospectivo, encontraron que los niveles eritrocíticos de $\mathrm{Pb}$ se asocian con ERC, los niveles de $\mathrm{Cd}$ incrementan el desarrollo de ERC, mientras que los niveles de mercurio no se asocian con ERC. Sin embargo, mencionan la necesidad de más estudios para evaluar la causalidad. Los análisis específicos de género sugieren posibles diferencias en la susceptibilidad o en la fiabilidad de la exposición al biomarcador.

$\mathrm{Ni}$ et al. $^{51}$ realizaron pruebas con células endoteliales coronarias y células de músculo liso vascular humanas cultivadas, en las que se investigaron especies reactivas de oxígeno (ROS), producidas por dichas células cuando son expuestas al plomo. Encontraron que la exposición al $\mathrm{Pb}$ promueve la generación de superóxido y peróxido de hidrógeno en las células endoteliales coronarias y células de músculo liso vascular humanas. Este fenómeno puede contribuir a la patogénesis de la hipertensión y las enfermedades cardiovasculares asociadas al $\mathrm{Pb}$. Los autores señalan, por tanto, el beneficio potencial de reducir la carga de $\mathrm{Pb}$ en las poblaciones expuestas (Tabla 2).

Tabla 2. Mecanismo de daño por intoxicación con metales pesados en humanos.

\begin{tabular}{|c|c|c|}
\hline Contaminante & Vía de entrada & Mecanismo de daño \\
\hline Cadmio & Gástrica & $\begin{array}{l}\text {-Disfunción por formación de radicales libres. } \\
\text {-Apoptosis: Activación por vía de las caspasas. } \\
\text {-Desnaturalización proteica. } \\
\text {-Disminución de la resistencia transepitelial. }\end{array}$ \\
\hline Plomo & Gástrica/Respiratoria/Contacto & $\begin{array}{l}\text {-Almacenamiento en hueso. } \\
\text {-Daño mitocondrial y formación de radicales libres. } \\
\text {-Apoptosis: Activación por vía de las caspasas. } \\
\text {-Inflamación por activación FN- } \kappa \beta \text {, SRAA y } \\
\text { atracción de macrófagos. } \\
\text {-Disminución de producción de NO. }\end{array}$ \\
\hline Arsénico & Gástrica/Respiratoria/Contacto & $\begin{array}{l}\text {-Depleción de GSH. } \\
\text { •Formación de radicales libres. } \\
\text { •Apoptosis: Activación por vía de las caspasas. }\end{array}$ \\
\hline Mercurio & Gástrica/Respiratoria/Contacto & $\begin{array}{l}\text {-Daño por reacción antígeno-anticuerpo específico. } \\
\text {-Depleción de función linfocitaria. }\end{array}$ \\
\hline
\end{tabular}

FN- $\kappa$ : Factor de necrosis Kappa Beta, SRAA: Sistema renina angiotensina aldosterona, NO: Óxido nítrico, GSH: Glutatión.

\section{Tratamiento}

Se carece, en gran medida, de medidas terapéuticas que disminuyan los efectos sistémicos de los diferentes contaminantes mencionados en esta revisión. En general, no existen tratamientos establecidos para disminuir los niveles de estos componentes en la sangre o para prevenir su toxicidad. Sin embargo, muchas terapias y alimentos funcionales han sido probados en animales con el fin de prevenir la nefrotoxicidad, por ejemplo, del arsénico. El más conocido es el resveratrol, que atenúa significativamente la acumulación de arsénico en los tejidos renales, disminuye los marcadores del estrés oxidativo y muestra menos lesiones morfológicas y necrosis tubular ${ }^{52}$. 
Asimismo, otros compuestos como la naringenina, una flavanona cítrica natural y el extracto de té verde han mostrado efectos promisorios que protegen al riñón contra la nefrotoxicidad ${ }^{53}$.

Por tal motivo, se necesita mayor vigilancia para disminuir las concentraciones ambientales de estos compuestos.

\section{Conclusiones}

En conclusión, los mecanismos fisiopatológicos de los contaminantes mencionados permiten, a través de su toxicidad, conocer los diferentes efectos a nivel renal. Un número creciente de evidencias, obtenidas tanto de humanos como de animales, sugiere que estos contaminantes son un factor de riesgo de morbilidad importante y que ocasionan, en la mayoría de los casos, alteraciones tubulares renales, específicamente en el túbulo contorneado proximal, y, algunas veces, falla renal aguda, que puede progresar a enfermedad renal crónica. Esta es una de las características clínicas asociadas con la exposición crónica. El plomo, principalmente, puede aumentar el riesgo de llegar a etapas terminales, aunque se necesitan más estudios para evaluar esta causalidad.

Debido a la alta presencia de estos contaminantes en el ambiente, así como a la carencia de medidas terapéuticas para menguar sus efectos, es necesario realizar más estudios longitudinales para evaluar las relaciones de las exposiciones a tales metales con el daño renal. De este modo, se podrían conocer los efectos crónicos y prestar una mayor vigilancia ambiental para disminuir las concentraciones de estos compuestos. Por ello, se requiere con urgencia disminuir las cantidades de metales pesados en la industria, la minería y el sector agropecuario, particularmente en los casos donde presten menor utilidad. Así, finalmente, consideramos pertinente la unión de esfuerzos entre el sector público y el privado, junto con centros de investigación, para adelantar trabajos que exploren e investiguen otras sustancias o metales menos tóxicos que puedan reemplazar o minimizar el impacto de los metales pesados descritos en este trabajo.

\section{Conflicto de intereses}

Los autores reportan que no existen conflictos de intereses.

\section{Financiación}

El presente trabajo de revisión no tuvo financiación alguna por parte de ningún organismo o empresa.

\section{Responsabilidades éticas}

\section{Protección de personas y animales}

Los autores declaran que para esta investigación no se han realizado experimentos en seres humanos ni en animales.

\section{Confidencialidad de los datos}

Los autores declaran que han seguido los protocolos de su centro de trabajo sobre la publicación de datos de pacientes.

\section{Derecho a la privacidad y consentimiento informado}

Los autores declaran que en este artículo no aparecen datos de pacientes.

\section{Contribución de los autores}

Juan Daniel Díaz García: contribuyó con la idea original y el proceso de realización de la revisión con respecto a la búsqueda de referencias, selección de la información, estructura de la revisión (resumen, introducción, desarrollo, conclusión, bibliografía, tablas y figuras) y cambios durante su proceso de publicación.

Emmanuel Arceo: contribuyó con la búsqueda y desarrollo de la revisión. 


\section{Referencias}

1. Ferraro PM, Costanzi S, Naticchia A, et al. Low-level exposure to cadmium increases the risk of chronic kidney disease: Analysis of the NHANES 1999-2006. BMC Public Health. 2010; 10:304.

2. Weaver VM, Kim NS, Jaar BG, et al. Associations of low-level urine cadmium with kidney function in lead workers. Occup Environ Med. 2011; 68:250-256.

3. Fujishiro H, Okugaki S, Kubota K, Fujiyama T, Himeno S. The role of ZIP8 down-regulation in cadmium-resistant metallothionein-null cells. J Appl Toxicol 2009; 29:367-73.

4. Thévenod F. Catch me if you can! Novel aspects of cadmium transport in mammalian cells. Biometals. 2010; 23:857-75.

5. Liu Y, Liu J, Klaassen CD. Metallothionein-null and wild-type mice show similar cadmium absorption and tissue distribution following oral cadmium administration. Toxicol Appl Pharmacol. 2001; 175:253-9.

6. Olivi L, Sisk J, Bressler J. Involvement of DMT1 in uptake of Cd in MDCK cells: role of protein kinase C. Am J Physiol Cell Physiol. 2001; 281:C793-800.

7. Hirano S, Sun X, DeGuzman C, Ransom R, MacLeish K, Smoyer WE, et al. p38 MAPK/ HSP25 signaling mediates cadmium-induced contraction of mesangial cells and renal glomeruli. Am J Physiol Renal Physiol. 2005; 288:F1133-43.

8. Gunawardana CG, Martínez RE, Xiao W, Templeton DM. Cadmium inhibits both intrinsic and extrinsic apoptotic pathways in renal mesangial cells. Am J Physiol Renal Physiol. 2006; 290:F1074-82.

9. Klaassen CD, Liu J, Diwan BA. Metallothionein protection of cadmium toxicity. Toxicol Appl Pharmacol 2009; 238:215-20.

10. Ekong EB, Jaar BG, Weaver VM. Lead-related nephrotoxicity: A review of the epidemiologic evidence. Kidney Int 2006; 70:207484.

11. Wang L, Wang H, Hu M, Cao J, Chen D, Liu Z. Oxidative stress and apoptotic changes in primary cultures of rat proximal tubular cells exposed to lead. Arch Toxicol. 2009;83:417-27.

12. Chiu TY, Teng HC, Huang PC, Kao FJ, Yang DM. Dominant role of Orai1 with STIM1 on the cytosolic entry and cytotoxicity of lead ions. Toxicol Lett. 2009;110:353-62.

13. Handlogten M, Shiraishi N, Awata H, Huang C, Tyler-Miller R. Extracellular Ca2-sensing receptor is a promiscuous divalent cation sensor that responds to lead. Am J Physiol Renal Physiol. 2000; 279:F1083-91.

14. Bravo Y, Quiroz Y, Ferrebuz A, Vaziri N, Rodríguez-Iturbe B. Mycophenolate mofetil administration reduces renal inflammation, oxidative stress, and arterial pressure in rats with lead-induced hypertension. Am J Physiol Renal Physiol. 2007;293:F616-23.

15. Vaziri N. Mechanisms of lead-induced hypertension and cardiovascular disease. Am J Physiol Heart Circ Physiol. 2008;295:H45465.

16. Courtois E, Marques M, Barrientos A, Casado S, López-Farré A. Lead-induced downregulation of soluble guanylate cyclase in isolated rat aortic segments mediated by reactive oxygen species and cyclooxygenase-2. J Am Soc Nephrol. 2003;14:1464-70.

17. Ni Z, Hou S, Barton C, Vaziri N. Lead exposure raises superoxide and hydrogen peroxide in human endothelial and vascular smooth muscle cells. Kidney Int. 2004;66:2329-36.

18. Thomas D. Unraveling arsenic-glutathione connections. Toxicol Sci 2009;107:309-11. Carbrey JM, Song L, Zhou Y, Yoshinaga M, Rojek A. Reduced arsenic clearance and increased toxicity in aquaglyceroporin-9-null mice. Proc Natl Acad Sci USA 2009; 106:15956-60.

19. Lee TC, Ho IC, Lu WJ, Huang JD. Enhanced expression of multidrug resistance-associated protein 2 and reduced expression of aquaglyceroporin-3 in an arsenic-resistant human cell line. J Biol Chem 2006;281:18401-7.

20. Gonick HC. Nephropathies in heavy metal intoxication. In: Massry SG, Glassock RJ. 4th Edición. Lippincott William \&Wilkins, Philadelphia 2001. Pp.933-934.

21. Hill G. Drug-associated glomerulopathies. Toxicol Pathol 1986; 14: 37-44.

22. Aymaz S, Gros O, Krakamp B, et al. Membranous nephropathy from exposure to mercury in the fluorescent-tuberecycling industry. Neprol Dial Transplant 2001; 16: 2253-2255.

23. Cameron JS, Trounce JR. Membranous glomerulonephritis and the nephrotic syndrome appearing during mersalyl therapy. Guys 
Hospi Rep 1965; 114: 101-107.

24. Chakera A, Lasseron D, Beck LH Jr, et al. Membranous nephropathy after use of UK-manufactured skin creams containing mercury. Q J Med 2011; 104: 893-896.

25. http://www.who.int/mediacentre/factsheets/fs361/en/WHO Mercury and health. Fact sheet $\mathrm{N}^{\circ} 361$.

26. Oliveira DBG, Foster G, Savill J, et al. Membranous nephropa- thy caused by mercury-containing skin lightening cream. Postgrad Med J 1987;63: 303-304.

27. El Azzouzi B, Tsangaris GT, Pellegrini O, Manuel Y, Benveniste J, and Thomas Y. Cadmium induces apoptosis in a human T cell line. Toxicology. 1994;88:127-139.

28. WC Prozialeck, VS Vaidya, J Liu, MP Waalkes, JR Edwards, PC Lamar, AM Bernard, X Dumont, and JV Bonventre. Kidney injury molecule-1 is an early biomarker of cadmium nephrotoxicity. Kidney Int. 2007 October; 72(8): 985-993.

29. Ziqiang Meng, Guohua Qin, Bo Zhang, Juli Bai. DNA damaging effects of sulfur dioxide derivatives in cells from various organs of mice. Mutagenesis. 2004;19(6):465-468.

30. P. C. Brazy, R. S. Balaban, S. R. Gullans, L. J. Nmiandel, V. AT. dennia. Relative effects of arsenate on sodium, phosphate, and glucose transport by the rabbit proximal tubule. J Clin Invest. 1980;66:1211-1221.

31. Jiangang Hou et al. 5-Aminolevulinic acid combined with ferrous iron induces carbon monoxide generation in mouse kidneys and protects from renal ischemia-reperfusion injury. Am J Physiol. 2013. 305:F1149-F1157.

32. Puri VN, Saha S. Comparison of acute cardiovascular effects of cadmium and captopril in relation to oxidant and angiotensin converting enzyme activity in rats. Drug Chem Toxicol.2003. 26(3):213-218.

33. Chen Y, Graziano JH, Parvez F, Liu M, Slavkovich V, Kalra T, et al. Arsenic exposure from drinking water and mortality from cardiovascular disease in Bangladesh: prospective cohort study. BMJ. 2011. 342:d2431; doi: 10.1136/bmj.d2431 [Online 5 May 2011].

34. Lewis DR, Southwick JW, Ouellet-Hellstrom R, et al. Drinking water arsenic in Utah: A cohort mortality study. Environ Health Perspect. 1999;107:359-365.

35. Meliker JR, Wahl RL, Cameron LL, et al. Arsenic in drinking water and cerebrovascular disease, diabetes mellitus, and kidney disease in Michigan: A standardized mortality ratio analysis. Environ Health. 2007;6:4.

36. Hsueh YM, Chung CJ, Shiue HS, et al. Urinary arsenic species and CKD in a Taiwanese population: A case-control study. Am J Kidney Dis. 2009; 54:859-870.

37. Bunderson M, Coffin JD, Beall HD. Arsenic induces peroxynitrite generation and cyclooxygenase-2 protein expression in aortic endothelial cells: possible role in atherosclerosis. Toxicol Appl Pharmacol. 2002; 184:11-18.

38. Carmignani M, Boscolo P, Castellino N. Metabolic fate and cardiovascular effects of arsenic in rats and rabbits chroni- cally exposed to trivalent and pentavalent arsenic. Arch Toxicol . 1985;(Suppl 8):452-455.

39. Chen Y, Factor-Litvak P, Howe GR, Graziano JH, Brandt-Rauf P, Parvez F, et al. Arsenic exposure from drinking water, dietary intakes of B vitamins and folate, and risk of high blood pressure in Bangladesh: a population-based, cross-sectional study. Am J Epidemiol . 2017; 165:541-552.

40. Druwe IL, Vaillancourt RR. Influence of arsenate and arsenite on signal transduction pathways: an update. Arch Toxicol. 2010; $84: 585-596$

41. Walton FS, Harmon AW, Paul DS, Drobna Z, Patel YM, Styblo M: Inhibition of insulin-dependent glucose uptake by trivalent arsenicals: possible mechanism of arsenic-induced diabetes. Toxicol Appl Pharmacol. 2004; 198:424-433.

42. Salazard B, Bellon L, Jean S, Maraninchi M, El Yazidi C, Orsiere T, Mar- gotat A, Botta A, Bergé-Lefranc J-L: Low-level arsenite activates the transcription of genes involved in adipose differentiation. Cell Biol Toxicol. 2004; 20:375-385.

43. Yoopan N, Watcharasit P, Wongsawatkul O, Piyachaturawat P, Satayavivad J. Attenuation of eNOS expression in cadmium-induced hypertensive rats. Toxicol Lett. 2008; 176(2):157-161.

44. Mueller PW, Price RG, Finn WF. New approaches for detecting thresholds of human nephrotoxicity using cadmium as an example. Environ Health Perspect. 106:227-230.

45. Lauwerys RR, Bernard AM, Roels HA, Buchet JP. Cadmium: exposure markers as predictors of nephro-toxic effects. Clin Chem 
40:1391-1394.

46. Roels H, Djubgang J, Buchet JP, Bernard A, Lauwerys R. Evolution of cadmium-induced renal dysfunction in workers removed from exposure. Scand J Work Environ Health.1982; 8:191-200.

47. Roels HA, Lauwerys RR, Buchets JP, Bernard AM, Vos A, Oversteyns M. Health significance of cadmium-induced renal dysfunction: a five-year follow up. Br J Ind Med. 1989; 46:755-764.

48. Mueller PW, Paschal DC, Hammel RR, Klincewicz SL, MacNeil ML, Spierto B, Steinberg KK. Chronic renal effects in three studies of men and women occupationally exposed to cadmium. Arch Environ Contam Toxicol. 1992;23:125-136.

49. Ja-Liang Lin, Dan-Tzu Lin-Tan, Kuang-Hung Hsu, Chun-Chen Yu. Environmental Lead Exposure and Progression of Chronic Renal Diseases in Patients without Diabetes. N Engl J Med. 2003;348:277-86.

50. Johan Nilsson Sommar, Maria K Svensson, Bodil M Björ, Sölve I Elmståhl, Göran Hallmans, Thomas Lundh, Staffan MI Schön, Staffan Skerfving, Ingvar A Bergdahl. End-stage renal disease and low-level exposure to lead, cadmium and mercury; a population-based, prospective nested case-referent study in Sweden. Environmental Health. 2013; $12: 9$.

51. Zhenmin Ni, Stephen Hou, Cyril H. Barton, Nosratola D. Vaziri. Lead exposure raises superoxide and hydrogen peroxide in human endothelial and vascular smooth muscle cells. Kidney International. 2004;66:2329-2336.

52. Yu M, Xue J, Li Y, et al. Resveratrol protects against arsenic trioxide-induced nephrotoxicity by facilitating arsenic metabolism and decreasing oxidative stress. Arch Toxicol. 2013; 87:1025-1035.

53. Bera AK, Rana T, Das S, et al. Mitigation of arsenic-mediated renal oxidative stress in rat by Pleurotus florida lectin. Hum Exp Toxicol. 2011; 30:940-951. 